\title{
Fundamental and subharmonic resonances of harmonically oscillation with time delay state feedback
}

\author{
A.F. EL-Bassiouny* \\ Department of Mathematics, Faculty of Science, Benha University, Benha 13518, Egypt
}

Received 16 May 2005

\begin{abstract}
Time delays occur in many physical systems. In particular, when automatic control is used with structural or mechanical systems, there exists a delay between measurement of the system state and corrective action. The concept of an equivalent damping related to the delay feedback is proposed and the appropriate choice of the feedback gains and the time delay is discussed from the viewpoint of vibration control. We investigate the fundamental resonance and subharmonic resonance of order one-half of a harmonically oscillation under state feedback control with a time delay. By using the multiple scale perturbation technique, the first order approximation of the resonances are derived and the effect of time delay on the resonances is investigated. The fixed points correspond to a periodic motion for the starting system and we show the external excitation-response and frequency-response curves. We analyze the effect of time delay and the other different parameters on these oscillations.
\end{abstract}

\section{Introduction}

The last decade has witnessed an increasing number of studies and applications of the active control of mechanical ad structural vibrations in various fields. Superior as the active control is over the passive control in many aspects, it involves more technical problems. One of the open problems is the complicated system dynamics induced by the unavoidale time delays in controllers and actuators, especially in various analogue filters, hydraulic and pneumatic actuators, where the time delays may give rise to the instability analysis of the controlled systems.

Nonlinear differential equations involving time delays have been studied in varies scientific fields, and some publications on this topic are cited in reference [1,2]. Active control of the motion of structures has recently received much attention (e.g, see reference [3,4]). Diekman et al. [5] performed a nonlinear analysis for determining Hopf bifurcations in systems of autonomous differential equations, while Belair and Camptell [6] extended this work to the case of multi-delayed differential equations. Palkovics and Venhovens [7] analyzed stability, Hopf bifurcations, and chaotic motion in controlled wheel suspension systems. Stepan and Haller [8] considered quasi-periodic oscillations in robot dynamics and Moiola et al. [9] dealt with the more general forced nonlinear system under delay control have been investigated by Plaut and Hsieh [10] in the case of nonlinear structural vibrations with a time delay in damping. $\mathrm{Hu}$ et al. [11] investigated the resonances of a harmonically forced Duffing oscillator with time delay state feedback. Using the method of multiple scales [12,13], they demonstrated that appropriate choices of feedback gain and the time delay are possible for a better vibration control. Asfar and Masoud [14] performed the control of parametric resonance using a Lanchester-type damper and obtain sucessful vibration suppression and bifurcations control. Thomsen [15] investigated a string with sliding-mass nonlinear absorber and showed that vibration suppression is possible for moderate forcing amplitudes. Hover, the system exhibits modulated responses, for high forcing amplitude or a large slider mass. Yabuno [16] proposed a control law based on linear velocity feedback and

*Correspondence to: Kingdom of Saudi Arabia, Quassim, Onaizah, P.O. Box 3137, Egypt. E-mail: atef elbassiouny@yahoo.com. 


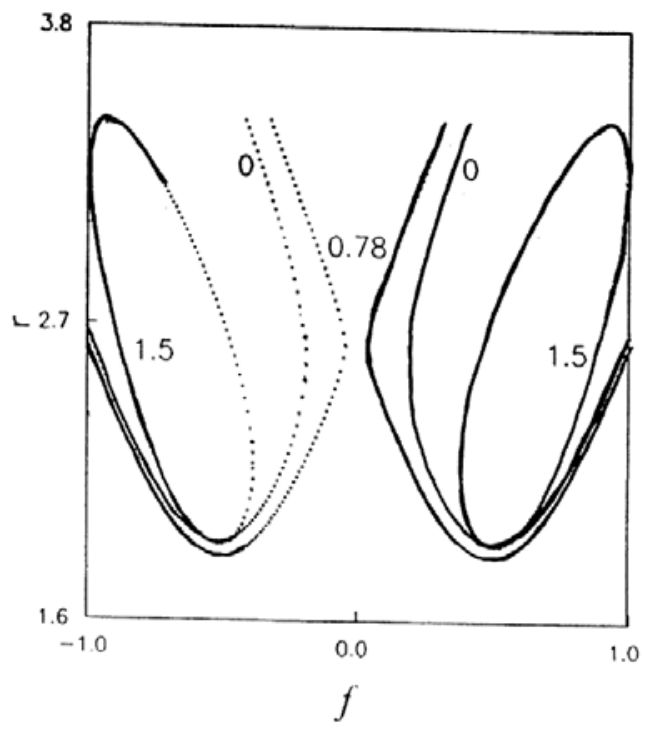

Fig. 1. External excitation-response curve for increasing the time delay $\tau$.

linear and cubic velocity feedback. His studies demonstrated that nonlinear position feedback reduces the response amplitude in the parametric excitation-response curve, while velocity feedback stabilizes the trivial solution in the frequency-response curve. Masccari [17] studied the parametric resonance of van der pol oscillator under state feedback control with time delay. Kouda and Mori [18] investigated the analysis of a ring of mutually coupled van der pol oscillators with coupling delay. Kouda and Mori [18] investigated the analysis of a ring of mutually coupled van der pol oscillators with coupling delay. Faria [19] investigated stability and bifurcation for a delayed predator for a delayed predator-prey model and effect of diffusion. Liao and Chen [20] studied local stability, Hopf and resonant codimension-two bifurcation in a harmonic oscillator with two time delays. Meng and Wei [21] analyzed stability and bifurcation of mutual system with time delay. Yuan and Han [22] investigated bifurcation analysis of a chemostat model with two distributed delays. Fofance and Ryba [23] investigated Parametric stability of nonlinear time delay equations. El-Bassiouny and Abdelhafez [25] analyzed the predication of bifurcations for external and parametric excited one-degree-of-freedom system with quadratic, cubic and quartic non-linearities. El-Bassiouny and Eissa [26] studied the dynamics of a single-degree-of-freedom structure with quadratic, cubic and quartic nonlinearities to a harmonic resonance. Elhefnawy and El-Bassiouny [27] analyzed the non-linear stability and chaos in electrohydrodynamics. El-Bassiouny [28] used the method of multiple scales to investigated principal parametric resonances of nonlinear mechanical system with two-frequency and self-excitations. Elnaggar and El-Bassiouny [29] studied harmonic, subharmonic, superharmonic, simultaneous sub/super-harmonic and combination resonances of self-excited second order system to multi-frequency excitations. Elnaggar and El-Bassiouny [30] investigated harmonic resonances of non-linear system of rods to a harmonic excitation. Maccari [31] analyzed Modulated motion and infinite-period bifurcation for two nonlinearly coupled and parametrically excited van der Pol oscillators. El-Bassiouny and Eissa [32] investigated resonance of non-linear systems subjected to multiparametrically excited structures. El-Bassiouny [33] analyzed the effect of non-linmearities in elastomeric material dampers on torsional oscillation control. Bi [34] studied the dynamical analysis of two coupled parametrically excited Van der pol oscillators.

The objective of this paper is to analyze the dynamics of a non-autonomous delay system. As the first study, a harmonically forced oscillation under linear time delay control is considered. This system serves as the simplest model for various controlled nonlinear systems, e.g., active vehicle suspension systems when the nonlinearity in tires is taken into acount [5]. The system motion is described by a second order nonlinear differential equation

$$
m \frac{d^{2} X(\bar{t})}{d \bar{t}^{2}}+c \frac{d X(\bar{t})}{d \bar{t}}+K X(\bar{t})+k_{1} X^{2}(\bar{t})+\mu k_{2} X^{3}(\bar{t})=\bar{u} X(\bar{t}-\bar{\tau})+\bar{v} \frac{d X(\bar{t}-\bar{\tau})}{d \bar{t}}+F \cos \omega t
$$




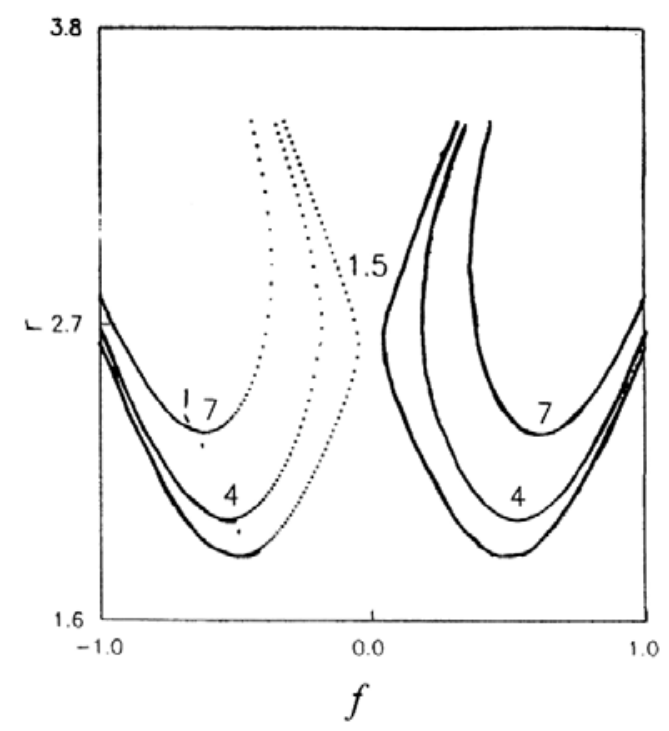

Fig. 2. External excitation-response curve for increasing the damping factor $\zeta$.

where $m>0, k_{1}>0, k_{2}>0$, and $0<\bar{\tau} \leqslant \frac{2 \Pi}{\omega}$. By using the following dimensionless time and new parameters

$$
\begin{aligned}
& t=\sqrt{\frac{k}{m}} \bar{t}, \quad \tau=\sqrt{\frac{k}{m}} \bar{\tau}, \quad u=\frac{\bar{u}}{2 k}, \quad v=\frac{\bar{v}}{2 \sqrt{m k}}, \\
& \zeta=\frac{c}{2 \sqrt{m k}}, \quad f=\frac{F}{k}, \quad \lambda=\omega \sqrt{\frac{m}{k}}
\end{aligned}
$$

Equation (1) can be written as

$$
\frac{d^{2} X(t)}{d t^{2}}+2 \zeta \frac{d X(t)}{d t}+X(t)+\gamma X^{2}(t)+\mu X^{3}(t)=2 u X(t-\tau)+2 v \frac{d X(t-\tau)}{d t}+f \cos \lambda t
$$

In the following sections, the fundamental resonance and subharmonic resonance of order one-half of a harmonically oscillation under state feedback control with a time delay will be studied by using the multiple scale perturbation technique

\section{Fundamental resonance}

\subsection{Steady-state resonance}

To investigate the fundamental resonance of the delay controlled system Eq. (3) by using the multiple scale perturbation technique, we confine the study to the case of small damping, week nonlinearities, week feedbak and soft excitation. That is

$$
\begin{aligned}
\zeta & =O(\varepsilon), & \gamma=O(\varepsilon), & \mu=O(\varepsilon), \\
f & =O(1), & & u=O(\varepsilon),
\end{aligned}
$$

where $\varepsilon \leqslant 1$ and $\sigma$ is the detuning parameter. Rewrite Eq. (3) as

$$
\frac{d^{2} X(t)}{d t^{2}}+X(t)=-2 \zeta \frac{d X(t)}{d \bar{t}}-\gamma X^{2}(t)-\mu X^{3}(t)+2 u X(t-\tau)+2 v \frac{d X(t-\tau)}{d t}+f \cos (1+\varepsilon \sigma) t
$$




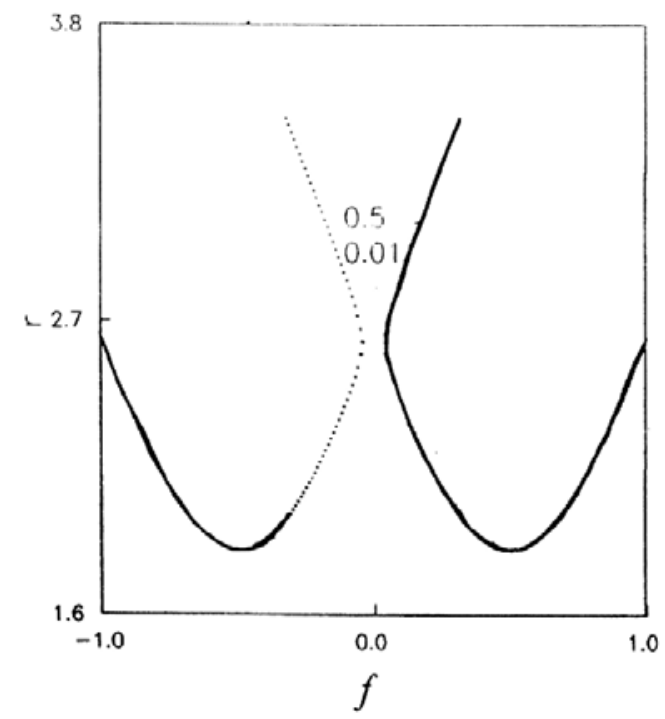

Fig. 3. External excitation-response curve for decreasing the damping factor $\zeta$.

We assume a two scale expansion of the solution

$$
X(t)=X_{0}\left(T_{0}, T_{1}\right)+X_{1}\left(T_{0}, T_{1}\right)+O\left(\varepsilon^{2}\right)
$$

where $T_{n}=\varepsilon^{n} t$. In terms of $T_{0}$ and $T_{1}$, the time derivative become

$$
\begin{aligned}
\frac{d}{d t} & =D_{0}+\varepsilon D_{1}+O\left(\varepsilon^{2}\right) \\
\frac{d^{2}}{d t^{2}} & =D_{0}^{2}+2 \varepsilon D_{0} D_{1}+O\left(\varepsilon^{2}\right)
\end{aligned}
$$

where $D_{n}=\frac{\partial}{\partial T_{n}}$. Inserting Eqs (6) and (7) into Eq. (5) and equating the coefficients of same power of $\varepsilon$, we obtain a set of linear partial differential equations

$$
\begin{aligned}
D_{0}^{2} X_{0}\left(T_{0}, T_{1}\right)+X_{0}\left(T_{0}, T_{1}\right)= & f \cos \left(T_{0}+\sigma T_{1}\right. \\
D_{0}^{2} X_{1}\left(T_{0}, T_{1}\right)+X_{1}\left(T_{0}, T_{1}\right)= & -2 D_{0} D_{1} X_{0}\left(T_{0}, T_{1}\right)-2 \zeta D_{0} X_{0}\left(T_{0}, T_{1}\right)-\gamma X_{0}^{2}\left(T_{0}, T_{1}\right)-\mu X_{0}^{3}\left(T_{0}, T_{1}\right) \\
& +2 u X_{0}\left(T_{0}-\tau, T_{1}\right)+2 v D_{0} X_{0}\left(T_{0}-\tau, T_{1}\right.
\end{aligned}
$$

The general solution of Eq. (8) can be expressed as

$$
X_{0}\left(T_{0}, T_{1}\right)=A\left(T_{1}\right) \exp \left(i T_{0}\right)+G \exp \left\{i\left(T_{0}+\sigma T_{1}\right)+c c\right.
$$

where $G=\frac{f}{2\left(1-\lambda^{2}\right)}$ and $c c$ denotes the complex conjugate of the preceding terms. Substituting Eq. (10) into Eq. (9), we obtain

$$
\begin{aligned}
D_{0}^{2} X_{1}\left(T_{0}, T_{1}\right)+X_{1}\left(T_{0}, T_{1}\right)= & -2 i\left(D_{1} A+\zeta A\right) \exp \left(i T_{0}\right)-\gamma\left(A^{2} \exp \left(2 i T_{0}\right)+2 \bar{A} G \exp \left\{(\lambda-1) T_{0}\right\}\right. \\
& +2 A \bar{A})-\mu\left(A^{3} \exp \left(3 i T_{1}\right)+3 A^{2} \bar{A} \exp \left(i T_{0}\right)\right)+2 A \bar{A} G \exp \left\{i\left(T_{0}+\sigma T_{1}\right)\right. \\
& +\bar{A} G^{2} \exp \left\{i\left(T_{0}+2 \sigma T_{0}\right)+2 i u A \exp \left(i T_{0}\right) \exp (-i \tau)+2 i v A \exp \left(i T_{0}\right)\right. \\
& \exp (-i \tau)+c c
\end{aligned}
$$

Then eliminating the secular terms from Eq. (11) yields

$$
-2 i\left(D_{1} A+\zeta A\right)-3 \mu A^{2} \bar{A}-2 \mu A \bar{A} G \exp \left(i \sigma T_{1}\right)-\mu \bar{A} G^{2}\left(2 i \sigma T_{1}\right)+2 i u A \exp (-i \tau)+2 i v A \exp (-i \tau)=0
$$




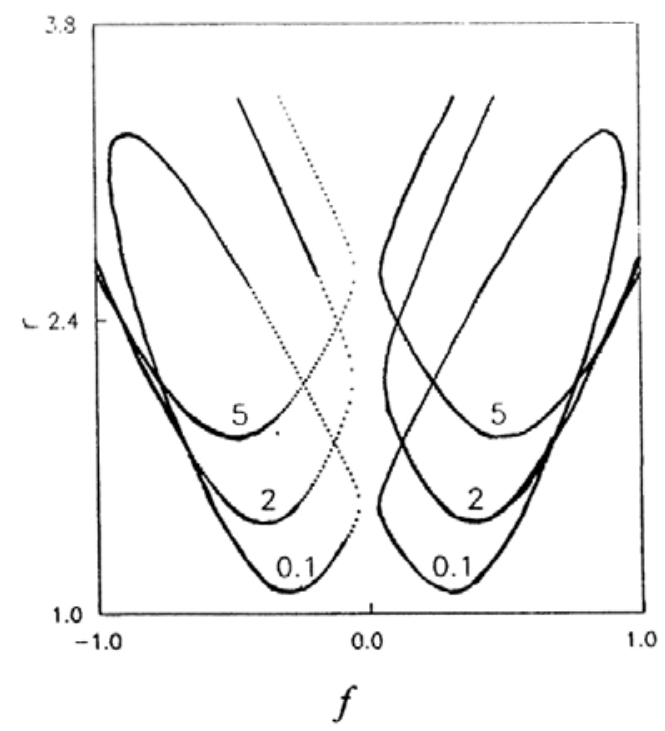

Fig. 4. External excitation-response curve for decreasing the detuming parameter $\sigma$.

It is convenient to put $A$ in the polar form

$$
A=\frac{1}{2} r\left(T_{1}\right) \exp \left\{i \beta\left(T_{1}\right)\right\}
$$

where $r$ and $\beta$ are the amplitude and phase. Substituting Eq. (13) into Eq. (12) and separating the real and imaginary parts, we obtain a set of autonomous differential equations that govern the amplitude $r\left(T_{1}\right)$ and the phase $\beta\left(T_{1}\right)$

$$
\begin{gathered}
D_{1} r=-\zeta r-\frac{1}{2} \mu r^{2} G \sin \phi-\frac{1}{2} \mu r G^{2} \sin 2 \phi-u r \sin \tau+v r \cos \tau \\
r D_{1} \beta=\frac{3}{8} \mu r^{3}+\frac{1}{2} \mu r^{2} G \cos \phi-\frac{1}{2} \mu r G^{2} \cos 2 \phi-u r \cos \tau-v r \sin \tau
\end{gathered}
$$

where

$$
\phi=\sigma T_{1}-\beta\left(T_{1}\right)
$$

From Eqs (14) and (15), we get a set of algebraic equations for amplitude rand phase $\phi$ of the steady-state fundamental resonance

$$
\begin{aligned}
& (\zeta+u \sin \tau-v \cos \tau) r=-\frac{1}{2} \mu r G(r \sin \phi+G \sin 2 \phi) \\
& (\sigma+u r \cos \tau+v r \sin \tau) r-\frac{3}{8} \mu r^{3}=-\frac{1}{2} \mu r G(r \cos \phi-G \cos 2 \phi)
\end{aligned}
$$

Equations (17) and (18) show that there are two possibilities: $r=0$ or $r \neq 0$. When $r \neq 0$, Eqs (17) and (18) become

$$
\begin{aligned}
& (\zeta+u \sin \tau-v \cos \tau)=-\frac{1}{2} \mu G(r \sin \phi+G \sin 2 \phi) \\
& (\sigma+u r \cos \tau+v r \sin \tau)-\frac{3}{8} \mu r^{2}=-\frac{1}{2} \mu G(r \cos \phi-G \cos 2 \phi)
\end{aligned}
$$

whereby we have the frequency response relation between $r$ and $\sigma$

$$
(\zeta+u \sin \tau-v \cos \tau)^{2}+\left((\sigma+u r \cos \tau+v r \sin \tau)-\frac{3}{8} \mu r^{2}\right)^{2}=\frac{1}{4} \mu^{2} G^{2}\left(r^{2}-G^{2}\right)
$$




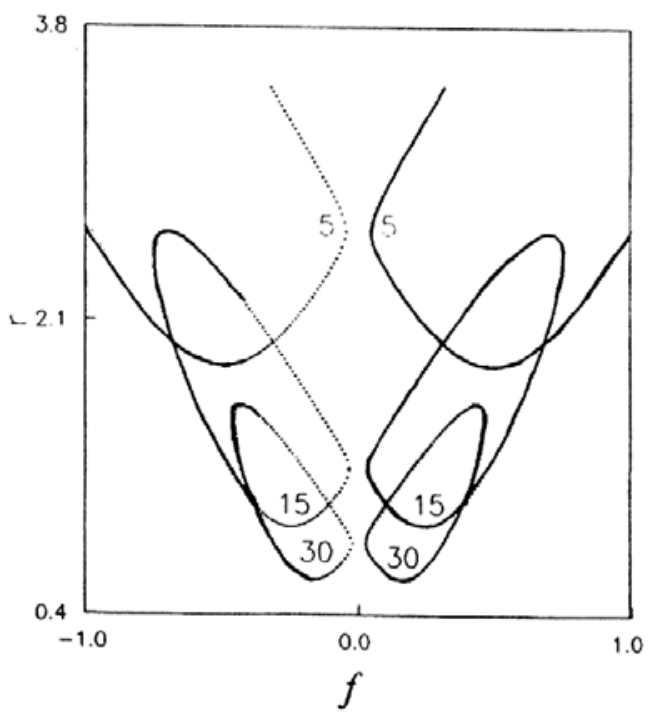

Fig. 5. External excitation-response curve for increasing the coefficient of cubic term $\mu$.

The first approximation for the fundamental resonance reads

$$
X(t)=r \cos (\lambda t-\phi)+\frac{f}{1-\lambda^{2}} \cos \lambda t+O(\varepsilon)
$$

\subsection{Stability analysis}

To determine the stability of the trivial solutions, one investigates the solutions of linearized form of Eq. (12); that is

$$
-2 i\left(D_{1} A+\zeta A\right)-\mu \bar{A} G^{2}\left(2 i \sigma T_{1}\right)+2 i u A \exp (-i \tau)+2 i v A \exp (-i \tau)=0
$$

\section{Letting}

$$
A=\left(B_{r}+i B_{i}\right) \exp \left(i \sigma T_{1}\right)
$$

in Eq. (23) where $B_{r}$ and $B_{i}$ are real, and separating real and imaginary parts, one obtains

$$
\begin{aligned}
& B_{i}^{\prime}+(\zeta+u \sin \tau-v \cos \tau) B_{i}=\left(\sigma-\frac{1}{2} \mu G^{2}+u \cos \tau+v \sin \tau\right) B_{r}=0 \\
& B_{r}^{\prime}+(\zeta+u \sin \tau-v \cos \tau) B_{r}-\left(\sigma+\frac{1}{2} \mu G^{2}+u \cos \tau+v \sin \tau\right) B_{i}=0
\end{aligned}
$$

Equations (25) and (26) admit solutions of the form

$$
\left(B_{r}, B_{i}\right)=\left(c_{1}, c_{2}\right) \exp \left(i s T_{1}\right)
$$

where $c_{1}$ and $c_{2}$ are arbitrary constants and

$$
s^{2}+2 m_{1} s+m_{1}^{2}+m_{2} m_{3}=0
$$

where 


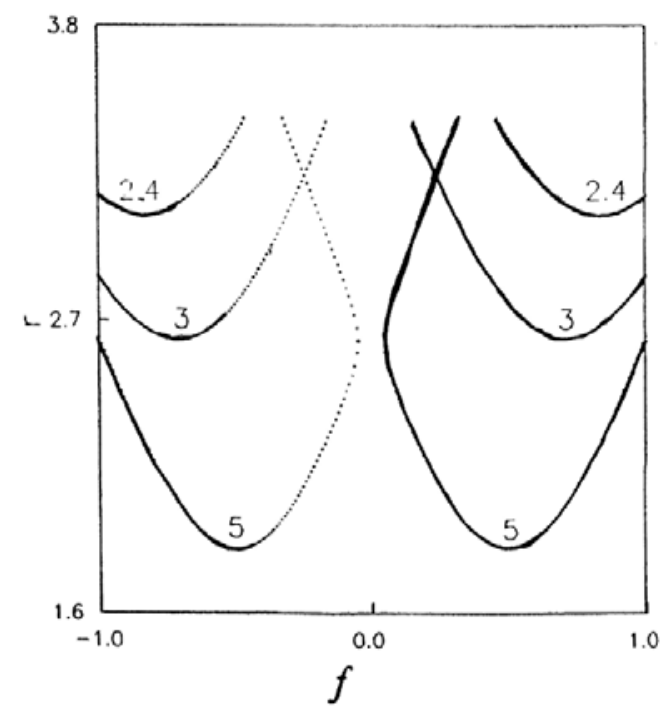

Fig. 6. External excitation-response curve for decreasing the coefficient of cubic term $\mu$.

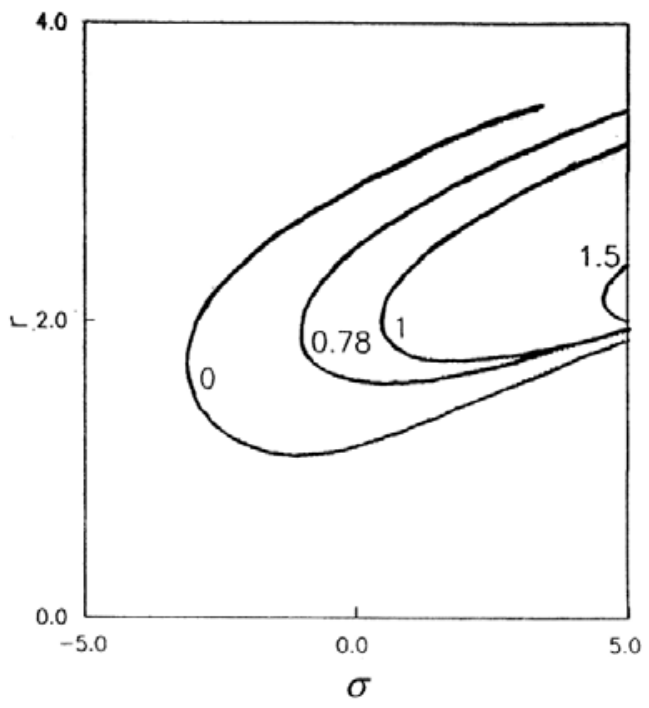

Fig. 7. Frequency-response curve for increasing the time delay $\tau$.

$$
\begin{aligned}
& m_{1}=(\zeta+u \sin \tau-v \cos \tau) \\
& m_{2}=\left(\sigma-\frac{1}{2} \mu G^{2}+u \cos \tau+v \sin \tau\right) \\
& m_{3}=\left(\sigma+\frac{1}{2} \mu G^{2}+u \cos \tau+v \sin \tau\right)
\end{aligned}
$$

From the Routh-Hurwitz criterion the steady-state vibration is asymptotically stable if and only if the following inequalities hold simultaneously

$$
\begin{aligned}
& m_{1}>0 \\
& \left(m_{1}^{2}+m_{2} m_{3}\right)>0
\end{aligned}
$$




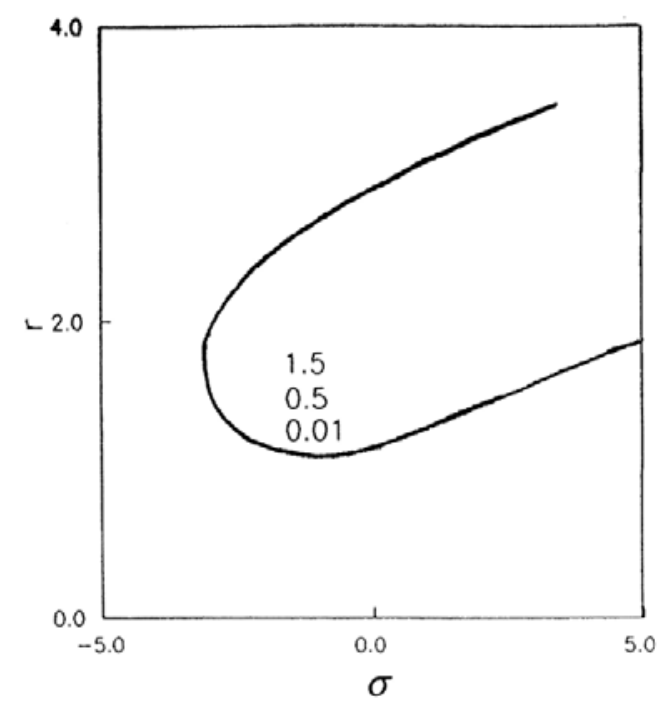

Fig. 8. Frequency-response curve for decreasing the damping factor $\zeta$.

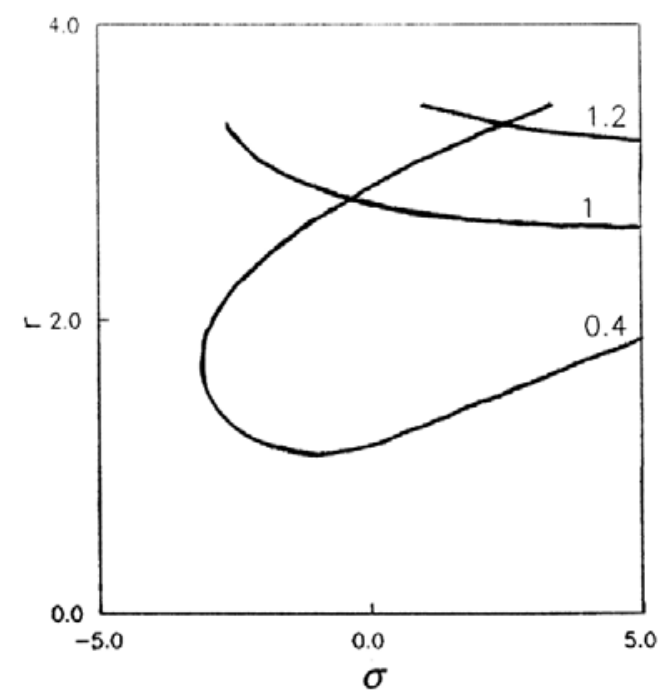

Fig. 9. Frequency-response curve for increasing the coefficient of external excitation $f$.

To analyze the stability of the non-trivial solutions, we linearize Eqs (14) and (15) at $(\hat{r}, \hat{\phi})$ with respect to $r$ and $\phi$

$$
\begin{aligned}
D_{1} \Delta r= & -\left[\zeta+\mu G \hat{r} \sin \hat{\phi}+\frac{1}{2} \mu G^{2} \sin 2 \hat{\phi}+u \cos \tau-v \sin \tau\right] \Delta r \\
& -\left[\frac{1}{2} \mu G \hat{r}^{2} \cos \hat{\phi}-\mu r G^{2} \hat{r} \sin 2 \hat{\phi}\right] \Delta \phi \\
D_{1} \Delta r=- & {\left[\frac{\sigma}{\hat{r}}-\frac{9}{8} \mu \hat{r}-\mu r G \cos \hat{\phi}+\frac{1}{2} \mu G^{2} \cos 2 \hat{\phi}+\frac{u}{\hat{r}} \cos \tau+\frac{v}{\hat{r}} \sin \tau\right] \Delta r } \\
& +\left[\frac{1}{2} \mu G \hat{r} \sin \hat{\phi}-\mu G^{2} \sin 2 \hat{\phi}\right] \Delta \phi
\end{aligned}
$$




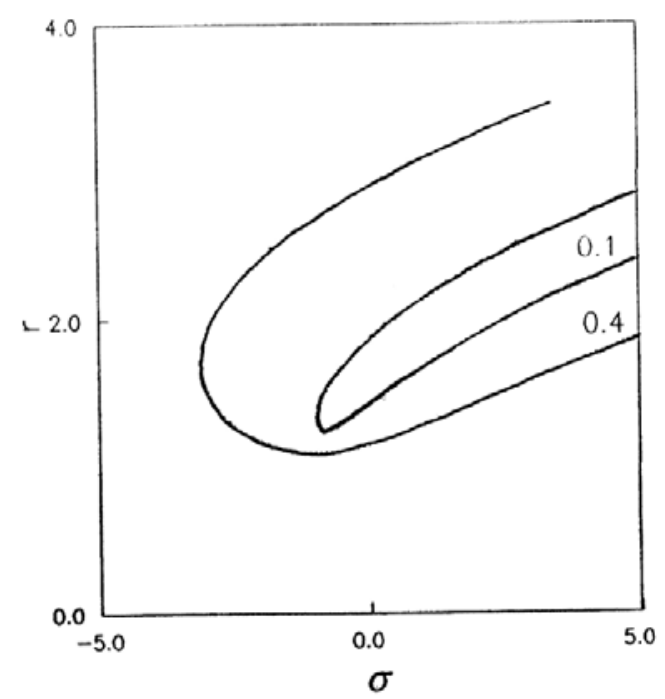

Fig. 10. Frequency-response curve for decreasing the coefficient of external excitation $f$.

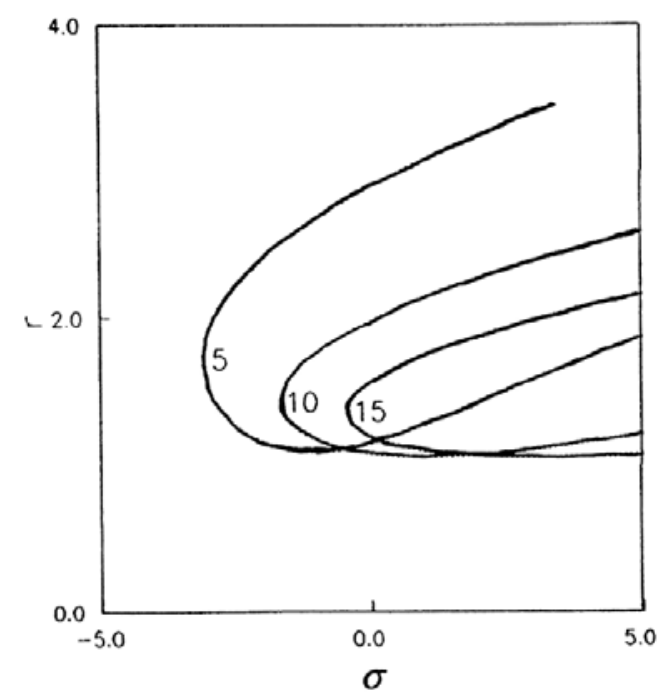

Fig. 11. Frequency-response curve for increasing the coefficient of cubic term $\mu$.

Equations (28) and (29) are linear equations with constants coefficients, and as such, posses a non-trivial solution proportional to $\exp \left(\Lambda T_{1}\right)$ if, and only if, the $\Lambda$ 's are the eigenvalues of the determinant of the coefficient matrix. If the real parts of all two $\Lambda$ 's are negative then the solution is considered stable.

\section{Principal resonance of order one-half}

\subsection{Steady-state resonance}

To study the principal harmonic resonance of order one-half of the controlled system, we confine ourselves to the case of 


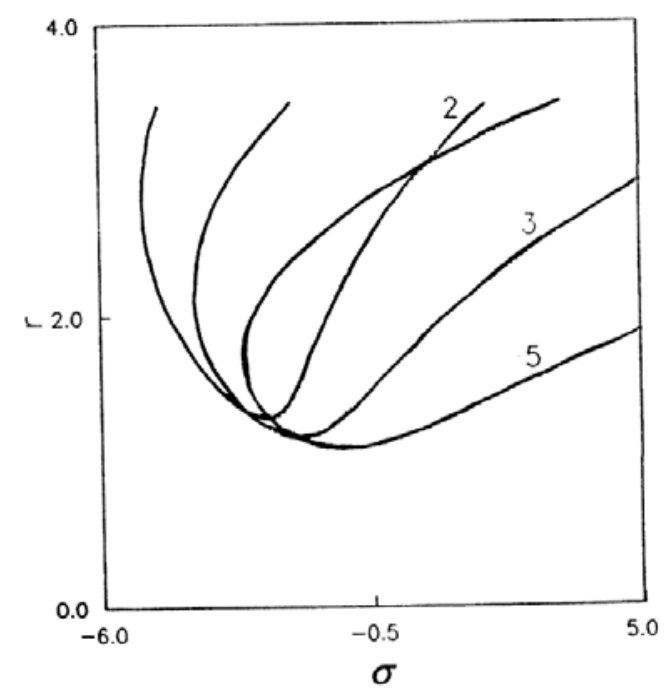

Fig. 12. Frequency-response curve for increasing the coefficient of cubic term $\mu$.

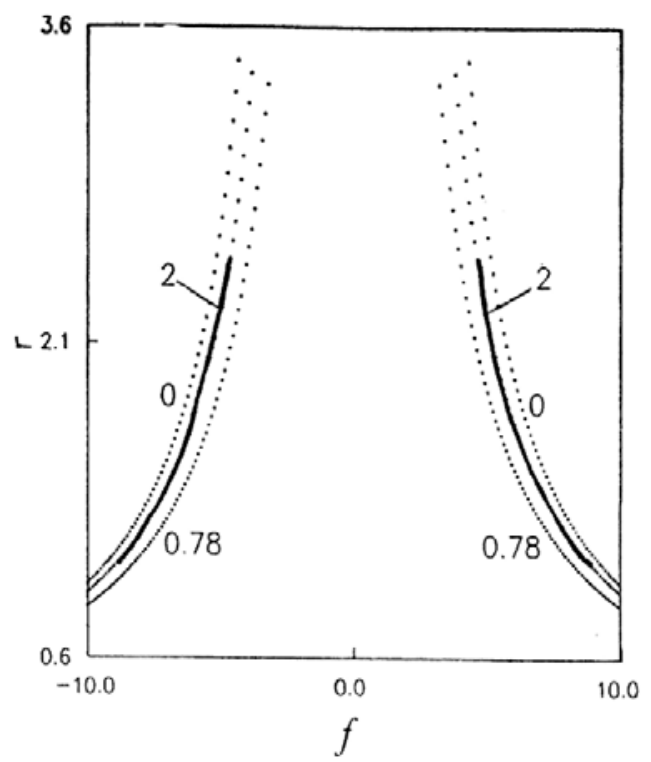

Fig. 13. External excitation-response curve for increasing the time delay $\tau$.

$$
\begin{aligned}
\zeta & =O(\varepsilon), & \gamma=O(\varepsilon), & \mu=O(\varepsilon), \\
f & =O(1), & & u=O(\varepsilon),
\end{aligned}
$$

Then, we rewrite Eq. (3) as

$$
\begin{aligned}
\frac{d^{2} X(t)}{d t^{2}}+X(t)= & -2 \zeta \frac{d X(t)}{d \bar{t}}-\gamma X^{2}(t)-\mu X^{3}(t)+2 u X(t-\tau) \\
& +2 v \frac{d X(t-\tau)}{d t}+f \cos \left(2 T_{0}+\sigma T_{1}\right)
\end{aligned}
$$

Inserting Eqs (6) and (7) into Eq. (34) and equating the coefficients of like power of $\varepsilon^{0}$ and $\varepsilon$, we obtain 


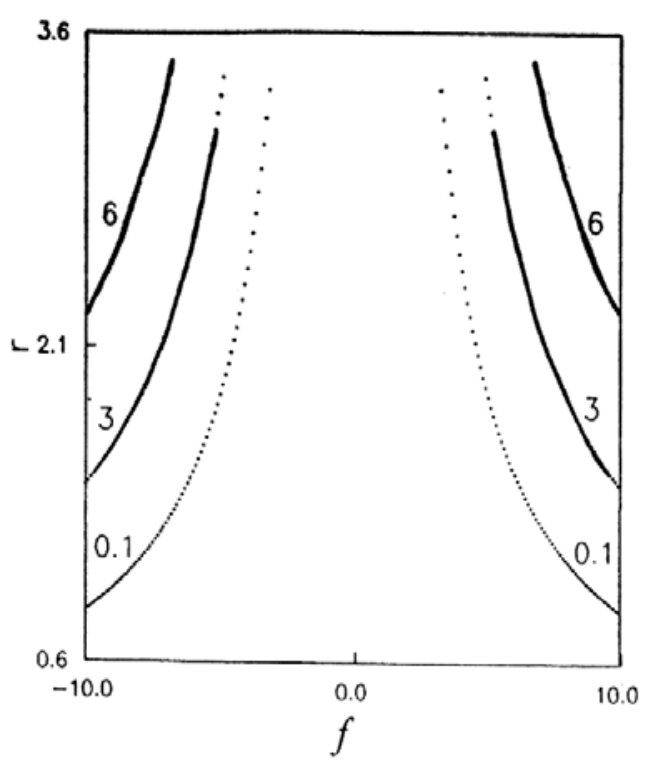

Fig. 14. External excitation-response curve for increasing the damping factor $\zeta$.

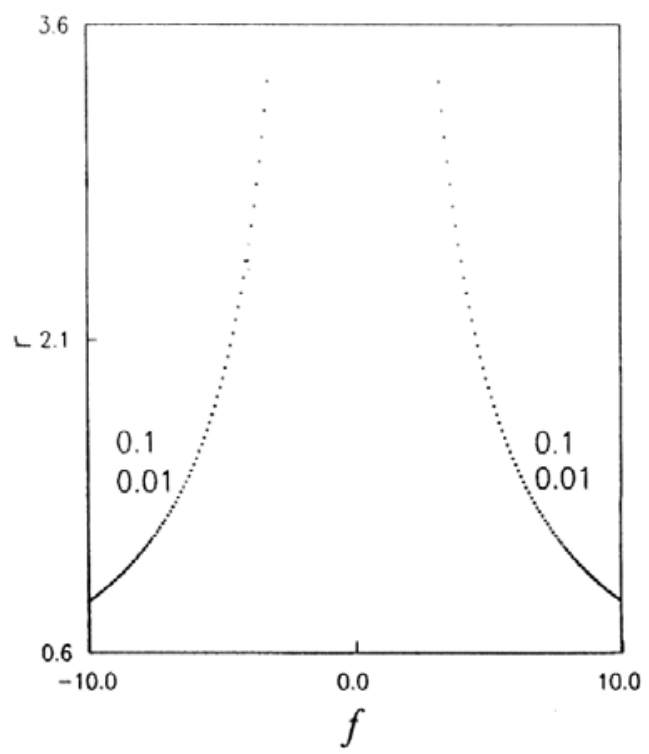

Fig. 15. External excitation-response curve for decreasing the damping factor $\zeta$.

$$
\begin{aligned}
D_{0}^{2} X_{0}\left(T_{0}, T_{1}\right)+X_{0}\left(T_{0}, T_{1}\right)= & f \cos \left(2 T_{0}+\sigma T_{1}\right) \\
D_{0}^{2} X_{1}\left(T_{0}, T_{1}\right)+X_{1}\left(T_{0}, T_{1}\right)= & -2 D_{0} D_{1} X_{0}\left(T_{0}, T_{1}\right)-2 \zeta D_{0} X_{0}\left(T_{0}, T_{1}\right)-\gamma X_{0}^{2}\left(T_{0}, T_{1}\right) \\
& -\mu X_{0}^{3}\left(T_{0}, T_{1}\right)+2 u X_{0}\left(T_{0}-\tau, T_{1}\right)+2 v D_{0} X_{0}\left(T_{0}-\tau, T_{1}\right)
\end{aligned}
$$

Solving Eq. (35) for $X_{0}\left(T_{0}, T_{1}\right)$, we have

$$
X_{0}\left(T_{0}, T_{1}\right)=A\left(T_{1}\right) \exp \left(i T_{0}\right)+G \exp \left\{i\left(2 T_{0}+\sigma T_{1}\right)+c c\right.
$$

Inserting Eq. (37) into Eq. (36) yields 


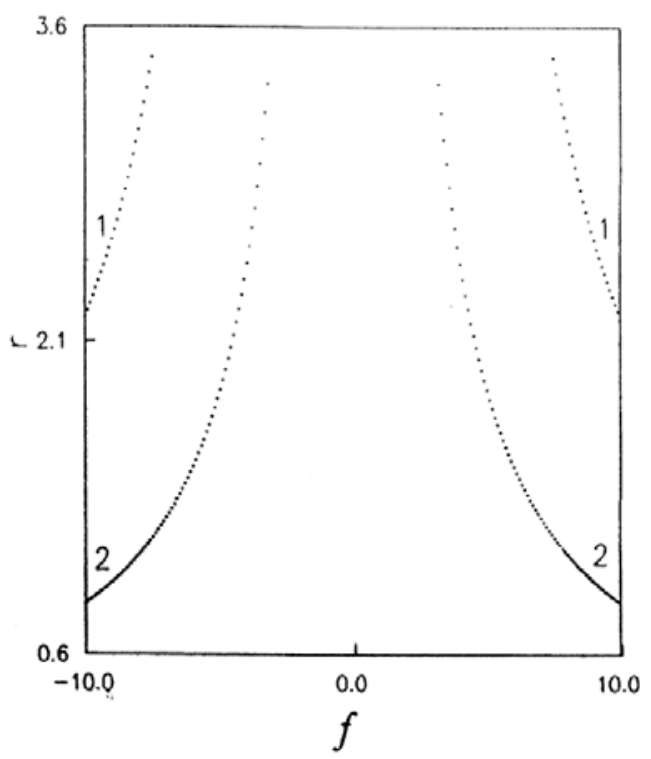

Fig. 16. External excitation-response curve for decreasing the coefficient of quadratic term $\gamma$.

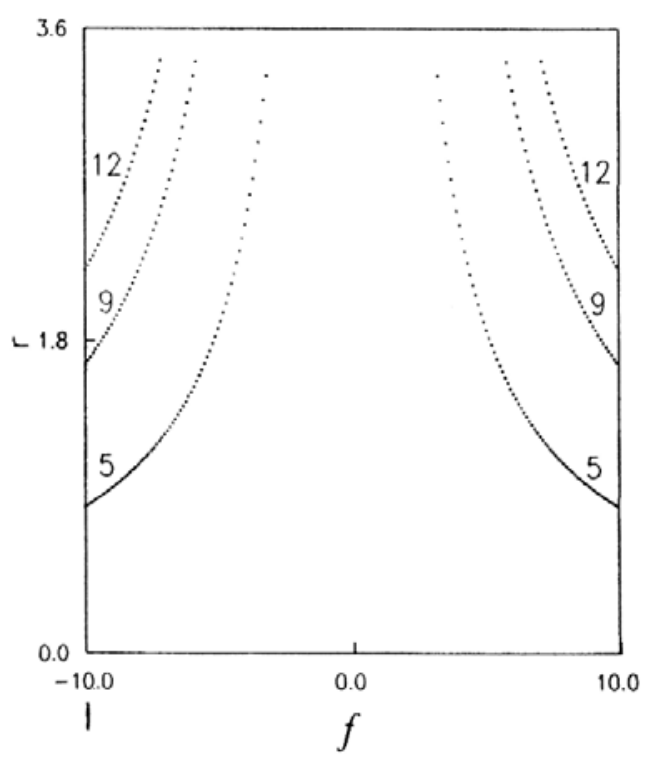

Fig. 17. External excitation-response curve for increasing the detuning parameter $\sigma$.

$$
\begin{aligned}
D_{0}^{2} X_{1}\left(T_{0}, T_{1}\right)+X_{1}\left(T_{0}, T_{1}\right)= & -2 i\left(D_{1} A+\zeta A\right) \exp \left(i T_{0}\right)-\gamma\left(A^{2} \exp \left(2 i T_{0}\right)+2 A \bar{A}+2 \bar{A} G \exp \right. \\
& \left\{i\left(T_{0}+\sigma T_{0}\right)\right)-\mu\left(A^{3} \exp \left(3 i T_{0}\right)+3 \mu A^{2} \bar{A} \exp \left(i T_{0}\right)\right) \\
& +2 i u A \exp \left(i T_{0}\right) \exp (-i \tau)+2 i v A \exp \left(i T_{0}\right) \exp (-i \tau)+c c
\end{aligned}
$$

The secular term of Eq. (11) vanishes if and only if

$$
-2 i\left(D_{1} A+\zeta A\right)-3 \mu A^{2} \bar{A}-2 \gamma \bar{A} G \exp \left(i \sigma T_{1}\right)+2 i u A \exp (-i \tau)+2 i v A \exp (-i \tau)=0
$$

Substituting Eq. (13) into Eq. (39) and separating the real and imaginary parts, we obtain the autonomous differential equations that govern the amplitude $r\left(T_{1}\right)$ and the phase $\beta\left(T_{1}\right)$ 


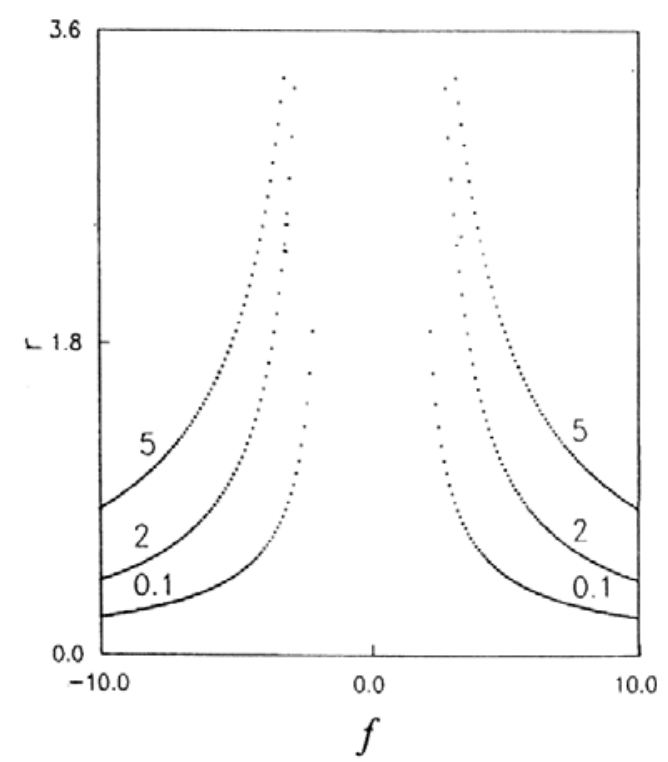

Fig. 18. External excitation-response curve for decreasing the detuning parameter $\sigma$.

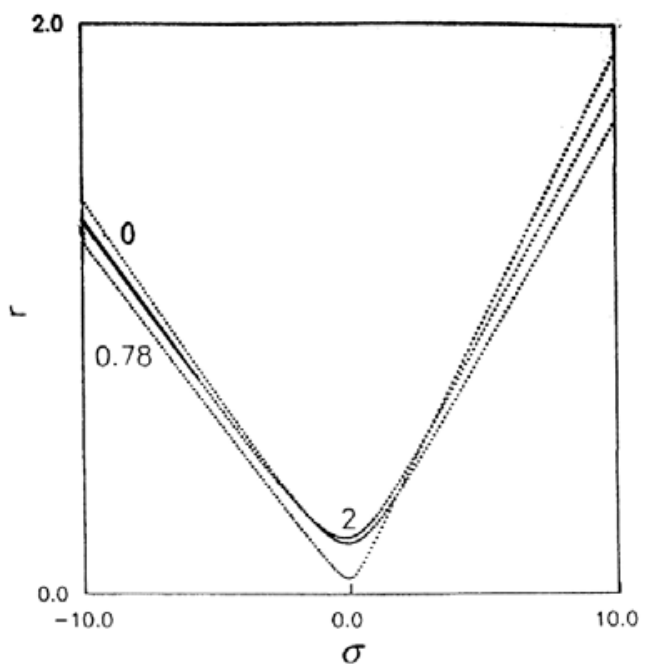

Fig. 19. Frequency-response curve for increasing the time delay $\tau$.

$$
\begin{aligned}
& D_{1} r=-\zeta r-\gamma r G \sin \phi-u r \sin \tau+v r \cos \tau \\
& r D_{1} \beta=\frac{3}{8} \mu r^{3}+\gamma r G \cos \phi-u r \cos \tau-v r \sin \tau
\end{aligned}
$$

where

$$
\phi\left(T_{1}\right)=\sigma T_{1}-2 \beta\left(T_{1}\right)
$$

From Eqs (40) and (41), we get a set of algebraic equations for amplitude $r$ and phase $\phi$ of the steady-state principal resonance of order one-half

$$
\begin{aligned}
& (\zeta+u \sin \tau-v \cos \tau) r=-\gamma r G \sin \phi \\
& \left(\frac{1}{2} \sigma+u \cos \tau+v \sin \tau\right) r-\frac{3}{8} \mu r^{3}=\gamma r G \cos \phi
\end{aligned}
$$




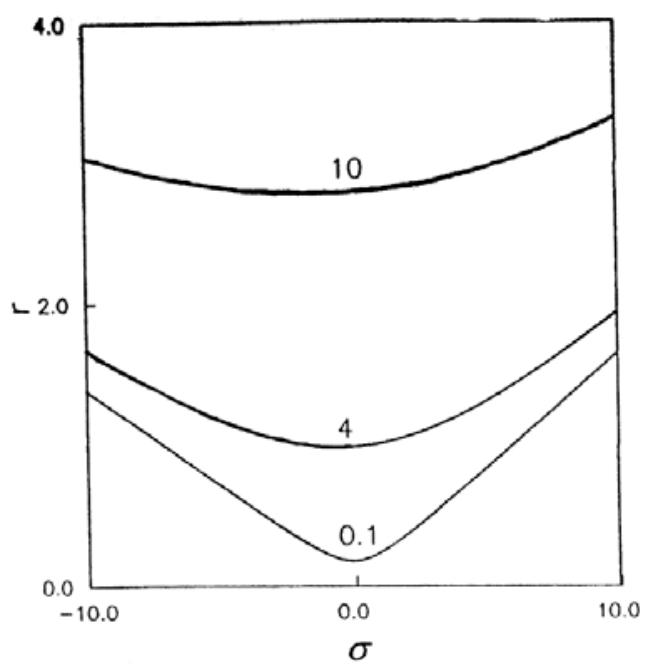

Fig. 20. Frequency-response curve for increasing the damping factor $\zeta$.

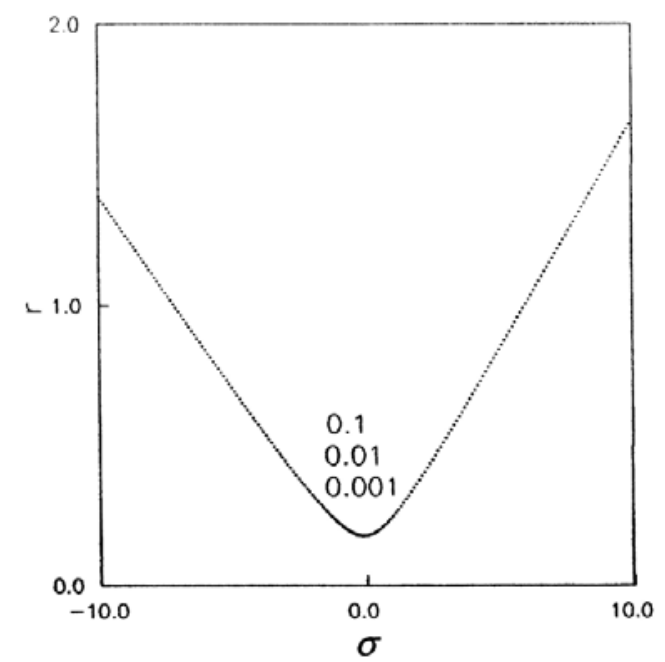

Fig. 21. Frequency-response curve for decreasing the damping factor $\zeta$.

Equations (43) and (44) show that there are two possibilities: $r=0$ (trivial solution) or $r \neq 0$ (non-trivial solution). When $r \neq 0$, we have

$$
\begin{aligned}
& (\zeta+u \sin \tau-v \cos \tau)=-\frac{1}{2} \gamma G \sin \phi \\
& \left(\frac{1}{2} \sigma+u r \cos \tau+v r \sin \tau\right)-\frac{3}{8} \mu r^{2}=\frac{1}{2} \gamma G \cos \phi
\end{aligned}
$$

whereby we derive the frequency response relation between $r$ and $\sigma$, and that between $\phi$ and $\sigma$

$$
\begin{aligned}
& (\zeta+u \sin \tau-v \cos \tau)^{2}+\left(\frac{1}{2} \sigma+u \cos \tau+v \sin \tau-\frac{3}{8} \mu r^{2}\right)^{2}-\gamma^{2} G^{2}=0 \\
& \tan \phi+\frac{\zeta+u \sin \tau-v \cos \tau}{\frac{1}{2} \sigma+u \cos \tau+v \sin \tau-\frac{3}{8} \mu r^{2}}=0
\end{aligned}
$$




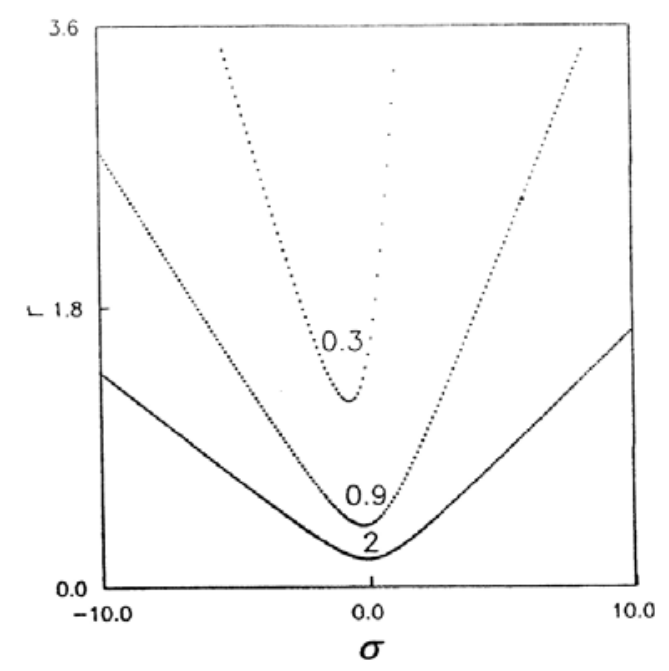

Fig. 22. Frequency-response curve for decreasing the coefficient of quadratic term $\gamma$.

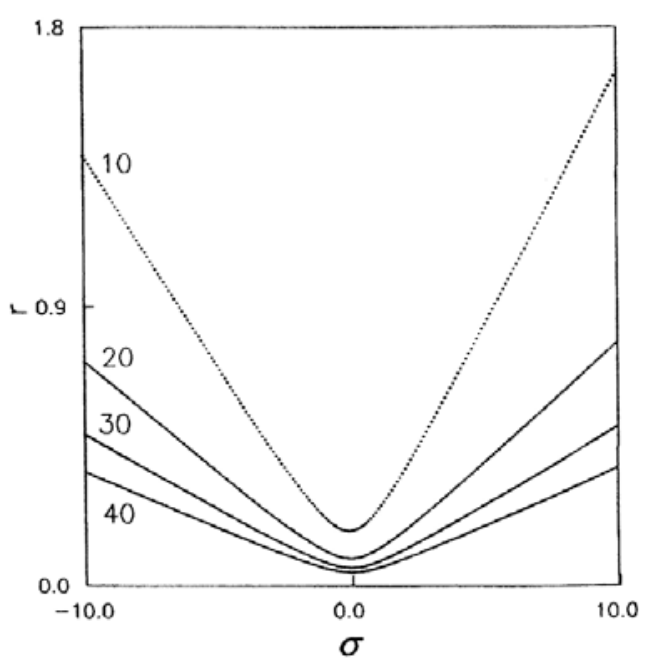

Fig. 23. Frequency-response curve for increasing the coefficient of external excitation $f$.

Then, the first-order approximation for the steady-state principal harmonic resonance of order one-half is given by

$$
X(t)=r \cos \left(\frac{\lambda t-\phi}{2}\right)+\frac{f}{1-\lambda^{2}} \cos \lambda t+O(\varepsilon)
$$

\subsection{Stability analysis}

To determine the stability of the steady state subharmonic resonance, we linearize Eqs (40) and (41) at $(\hat{r}, \hat{\phi})$ with respect to $r$ and $\phi$

$$
\begin{aligned}
& D_{1} \Delta r=[-\zeta-\gamma G \sin \hat{\phi}-u \sin \tau+v \cos \tau] \Delta r-(\gamma G \hat{r} \cos \hat{\phi}) \Delta \phi \\
& D_{1} \Delta r=\left[\frac{\sigma}{\hat{r}}-\frac{9}{4} \mu \hat{r}-\frac{2 \gamma G}{\hat{r}} \cos \hat{\phi}+\frac{2 u}{\hat{r}} \cos \tau+\frac{2 v}{\hat{r}} \sin \tau\right] \Delta r+2(\gamma G \cos 2 \hat{\phi}) \Delta \phi
\end{aligned}
$$




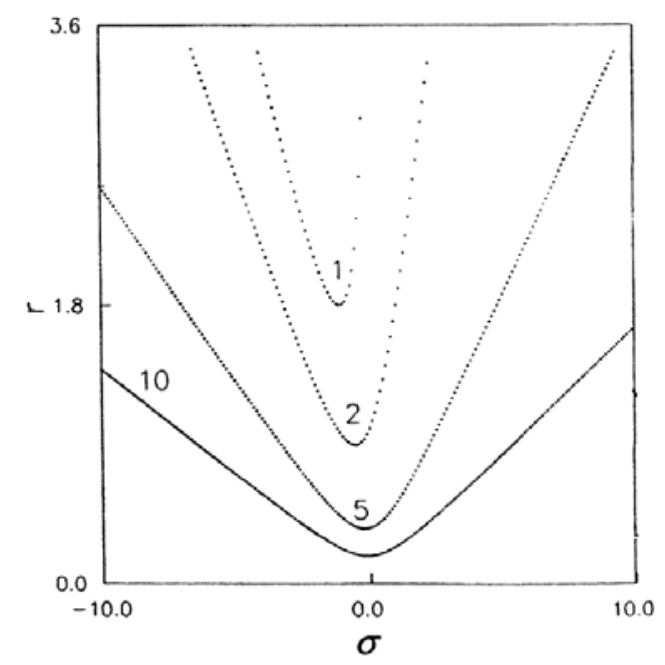

Fig. 24. Frequency-response curve for decreasing the coefficient of external excitation $f$.

The stability of a particular fixed point with respect to an infinitesimal disturbance proportional to $\exp \left(\Lambda T_{1}\right)$ is determined by the eigenvalues of the Jacobian matrix of the right hand of Eqs (50) and (51). A given fixed point is stable if and only if the real parts of all eigenvalues are less than or equal to zero. Note that the stability of the trivial solutions in this case is determined by using Eqs (50) and (51) when $\mu=0$.

\section{Numerical results and discussion}

The analytical analysis are represented graphically by using numerical method. The frequency Eqs (21) and (47) are nonlinear algebraic equation in the amplitude $(r)$. These equations are solved by using bisection method. The numerical results are plotted in a group of Figs 1-24, which represent the variation of the response amplitude $(r)$ with the coefficient of external excitation $f$ in Figs 1-6 for the first case and Figs 13-18 for the second case and represent variation of the amplitude $(r)$ with the detuning parameter $\sigma$ in Figs 7-12 for the first case and Figs 19-24 for the second case. The stability of a fixed-point solution is studied by examination of the eigenvalues of the Jacobian matrix of Eqs (14) and (15) for fundamental resonance and Eqs (40) and (41) equations for subharmonic resonance evaluated at the fixed point of interest. If all the eigenvalues have negative real parts, then the fixed point is expected to settle to it. These solutions are called stable nodes and denoted by solid lines in the frequency-response curves of Fig. 1 through 28. If a pure-real eigenvalue becomes positive, the fixed point loses stability and the motion is expected to diverge from it. These unstable solutions are called saddles and are denoted by dotted lines in Fig. 1 through 28. Thus, stable and unstable non-trivial solutions may be represented on the response curves by solid and dotted lines respectively.

Figures 1-6 show the external excitation-response curve for the variation of the parameters $\tau, \zeta, \sigma$, and $\mu$ in the case of fundamental resonance and these figures represented the variation of the response amplitude $(r)$ with the coefficient of external excitation $f$. Figure 1, represent the variation of the time delay $\tau$. As $\tau=0$, the amplitude $r$ has two semi-ovals which are opened from the upper and symmetric about $f=0$. The left semi-oval has stable and unstable solutions. As $f$ increases with negative values, left semi-oval loses stability via a saddle-node bifurcation at $f=0.4$. Also the right semi-oval has stable solutions. As $f$ increases with negative values, left semi-oval loses stability via a saddle-node bifurcation at $f=0.44$. Also the right semi-oval has stable solutions. When the time delay $\tau$ is increased further, there exists a contraction in the two semi-ovals and the zone of definition is decreased. For further increasing of the time delay $\tau$ up to 2, the amplitude $r$ has symmetric two oval about $f=0$ with decreasing in the zone of definition and the left oval has two bifurcation points and the right oval has stable solutions. Figures 2 and 3 represent the variation of increasing and decreasing the damping factor $\zeta$. For increasing damping factor $\zeta$ respectively, the two semi-oval are moved to the upper with increasing magnitudes. The 
regions of stability and definition are decreased and each left semi-ovals has one saddle-node bifurcation, Fig. 2. In Fig. 3, we note that the response amplitude does not affected with decreasing the damping factor $\zeta$ respectively and the region of stability is decreased. When $f$ increases with negative values, left semi-oval loses stability via a saddle-node bifurcation at $f=0.3$ and the right semi-oval has stable solutions. Figure 4 represents the variation of increasing the detuning parameter $\sigma$. When $\sigma$ is increased, we observed that the two symmetric semi-oval are moved to the down and have decreased magnitudes respectively. As $f$ increases with negative values, left semi-oval loses stability via a saddle-node bifurcation and the right semi-oval has stable solutions. For further decreasing of $\sigma$ up to 0.1 , the response amplitude $(r)$ has two symmetric ovals with decreased magnitudes and the region of multivalued is increased. The left oval has two saddle-node bifurcations and the right oval has stable solutions. For increasing the coefficient of cubic term $\mu$, the response amplitude $r$ has two symmetric ovals and have decreasing magnitudes and each oval intersect the semi-oval in two points. The left oval has two saddle-node bifurcations and the right oval has stable solutions. As $\mu$ increases further up to 30, the two ovals are contraction and moved to the down with decreasing magnitudes and the regions of definition and multivalued are decreased. The left oval has two saddle-node bifurcations and the right oval has stable solutions, Fig. 5. When the coefficient of cubic term $\mu$ is decreasing respectively, we note that the two symmetric semi-ovals are moved to the upper with increasing magnitudes and the region of definition is decreased, As $f$ increases with negative values, we observe that each left semi-ovals loses stability via a saddle-node bifurcation and each right semi-oval has stable solutions. Figure 6.

Figures 7-12 show the frequency-response curve for the variation of the parameters $\tau, \zeta, f$, and $\mu$ in the case of fundamental resonance and these figures represent the variation of the amplitude $(r)$ with the detuning parameter $\sigma$. Figure 7, represents the variation of the time delay $\tau$. When $\tau=0$, the response amplitude $r$ has multivalued continuous curve represented by semi-oval. The solutions are stable. For further increasing of $\tau$ respectively (i.e. $\tau$ take the values $0.78,1$, and 1.5 ), we note that the semi-oval is contraction respectively and the lower and the upper branches of the semi-oval have increased and decreased magnitudes respectively. The zone of definition is decreased respectively. Figure 7. For decreasing the damping factor $\zeta$ respectively, we observed that the response amplitude $r$ does not affected and the solutions are stable, Fig. 8. Figures 9 and 10 represent the variation of increasing the coefficient of external excitation $f$. As $f$ increasing respectively. The response amplitude $r$ moves to the upper and has stable single-valued curve. When $f$ is increased further, the single-valued curve moved to the upper and the region of definition is decreased, Fig. 9. As $f$ increases, the semi-oval is contraction and the zones of definition and multivalued are decreased, Fig. 10. When coefficient of cubic term $\mu$ is increased respectively, the semi-oval is contraction and moved to the down and the regions of multi-valued and definition are decreased, Fig. 11. For decreasing the coefficient of cubic term $\mu$ respectively, the multivalued is decreased and the zones of stability and definition are increased respectively, Fig. 12.

Figures 13-18 show the external excitation-response curves for the variation of the parameters $\tau, \zeta, \gamma$, and $\sigma$ in the case of subharmonic resonance and these figures represented the variation of the response amplitude $(r)$ with the coefficient of external excitation $f$. Figure 13, represent the variation of the time delay $\tau$. As $\tau=0$, the response amplitude $r$ has two single-valued curves which are symmetric about $f=0$ and the solutions are unstable. When the time delay $\tau$ is increased further up to 0.78 , the two single-valued curves have decreased unstable magnitudes. For further increasing of the time delay $\tau$ up to 2, the single-valued curves have increasing magnitudes and there exist stable and unstable solutions and both left and right branches have saddle-node bifurcations. Figures 14 and 15 represent the variation of increasing and decreasing the damping factor $\zeta$. As $\zeta$ increases, the two branches moves to the upper with increasing magnitudes and the zones of stability and definition are increasing and decreasing respectively. As $f$ increases with negative values, the left branch loses stability via a saddle-node bifurcation. Also when $f$ increases with positive values, the right branch loses stability via a saddle-node bifurcation. When $\zeta$ increases further up to 6 , the two single-valued curves moved to the upper with increasing stable magnitude. The zone of definition is decreased and the zone of definition is decreased, Fig. 14. For decreasing $\zeta$ respectively, the two single-valued curves do not affected and the solutions are unstable, Fig. 15. When the coefficient of quadratic term $\gamma$ is decreased respectively, the two curves move to the down with decreasing unstable magnitudes and the region of definition is increased, Fig. 16. As the detuning parameter $\sigma$ is increased respectively, the two symmetric curves move to the upper with unstable increasing magnitudes respectively and the zones of definition are decreased respectively, Fig. 17. For decreasing the detuning parameter $\sigma$ respectively, we get the same variation as in Fig. 4 such that all the solutions are unstable, Fig. 18. 
Figures 19-24 show the frequency-response curve for the variation of the parameters $\tau, \zeta, \gamma$, and $f$ in the case of subharmonic resonance and these figures represent the variation of the amplitude $(r)$ with the detuning parameter $\sigma$. Figure 19, represent the variation of the time delay $\tau$. When $\tau=0$, the response amplitude $r$ has single-valued curve which consisting of two branches. As $\sigma$ increases from negative value $(-10)$ respectively, we note that the response amplitude $r$ has decreased unstable magnitudes until it reach the value $\sigma=0$ and as $\sigma$ increases respectively, the response amplitude $r$ has increased unstable magnitudes respectively. When $\tau=0.78$, response amplitude $r$ moves to the upper with increased unstable magnitudes for the first branch in the interval [ $-10-2.5]$ and after this interval it has decreased unstable magnitudes. For further increasing of the time delay $\tau$ up to 2, the response amplitude $r$ has stable solutions through the interval $[(-10)-(-5.5)]$ and after this interval the solutions are unstable. Figures 2 and 3 , represent the variation of increasing and decreasing the damping factor $\zeta$. When $\zeta$ is increased, the singlevalued curve moved to the upper with increasing magnitudes and as $\sigma$ is increased from $(-10)$, the single-valued curve loses stability via a saddle-node bifurcation. When $\zeta$ increases further, the single-valued curve moved to the upper with increasing stable magnitudes, Fig. 20. From Fig. 21, we observe that, the single-valued curve does not affected by decreasing the factor $\zeta$ respectively and the solutions are unstable. As the coefficient of quadratic term $\gamma$ increases respectively, the single-valued curve moved to the upper with increasing unstable magnitudes and the zone of definition is decreased respectively, Fig. 22. Figures 23 and 25 represent the variation of increasing the coefficient of external excitation $f$. When $f$ is increased respectively. the single-valued curve moved to the lower with decreasing unstable magnitudes, Fig. 23. For increasing $f$ respectively, we get the same variation as in Fig. 22, Fig. 24.

\section{Concluding remarks}

The time delay in feedback control results in an infinite dimension for the controlled nonlinear system, and increases dramatically the complexity of the numerical analysis for the system dynamics. The method of multiple scales proves to be a powerful tool to gain insight into the fundamental and principal resonance of the system has one-degree-of-freedom with weak nonlinearities and weak delay feedback. We have derived two-slow-flow equations, governing the amplitude and phase of approximate long-time response of the oscillator. Determination of various types of steady state motion is then reduced to solution of sets of algebraic equations. Condition for existence of stable solution is determined. Solution of the frequency-response equation for the two cases shows the following conclusions:

- The external excitation-response curves for the two cases are symmetric about $f=0$.

- The external excitation-response curves does not affected by decreasing the damping factor.

- In the case of fundamental resonance, we note that the right branches have stable solutions.

- The frequency-response curves have stable solutions for the variation of the parameters $\tau, \zeta, f$ and $\mu$.

- The frequency- response curves for the two cases does not affected by decreasing the damping factor.

\section{References}

[1] Y. Kuang, Delay differential equations with applications in population dynamics, New York, Academic Press, 1993.

[2] J. Hall, Theory of differential equations, Springer-Verlag, New York, 1997.

[3] K. Gopalsamy, Stability and oscillations in delay differential equations of population Dynamics, Kluwer Dordrecht, 1992.

[4] Y. Kuang, Delay differential equations with application in population dynamics, New York: Springer, 2002.

[5] O. Diekmann, S.A. Van Gils, S.M. Verduyn Lunel and H.O. Walther, Delay equations, functional, complex, and nonlinear analysis, Springer-Verlag, New York, 1995.

[6] J. Belair and S.A. Cambell, Stability and bifurcation of equilibria in a multi-delayed differential equation, SIAM Journal on Applied Mathematics 54(5) (1994), 1402-1424.

[7] L. Palkovics and P.J. Venhovens, Investigation on stability and possible chaotic motion in the controlled wheel suspension system, Vehicle System Dynamics 21(5) (1992), 269-296.

[8] G. Stepan and G. Haller, Quasiperiodic oscillations in robot dynamics, Nonlinear Dynamics 8(4) (1995), 513-528.

[9] J.L. Moiola, H.G. Chiacchiarini and A.C. Desages, Bifurcations and Hopf degeneracies in nonlinear feedback systems with time delay, International Journal of Bifurcation and Chaos 64(4) (1996), 661-672. 
[10] R.H. Plaut and J.C. Hsieh, Non-linear structural vibrations involving a time delay in damping, Journal of Sound and Vibration 117(3) (1987), 497-510

[11] H. Hu, E.H. Dowell and L.N. Virgin, Resonances of a harmonically forced Duffing oscillator with time delay state feedback, Nonlinear Dynamics 15(4) (1998), 311-327.

[12] A.H. Nayfeh and D.T. Mook, Nonlinear Oscillations, Wiley, New York, 1979

[13] A.H. Nayfeh, Perturbation Techniques, Wiley, New York, 1981.

[14] K.R. Asfar and K.K. Masoud, Damping of parametrically excited single-degree-of-freedom systems, International Journal of Non-linear Mechanics 29 (1994), 421-428.

[15] J.J. Thomsen, Vibration suppression by using self-arranging mass: Effects of adding restoring force, Journal of Sound and Vibration 197 (1996), 403-425.

[16] H. Yabuno, Bifurcation control of parametrically excited Duffing systems by a combined linear-plus-nonlinear feedback control, Nonlinear Dynamics 12 (1997), 236-274.

[17] A. Maccari, The response of a parametrically excited van der Pol oscillator to a time delay state feedback, Nonlinear Dynamics 26 (2001), $105-119$.

[18] A. Kouda and S. Mori, Analysis of a ring of mutually coupled van der pol oscillators with coupling delay, IEEE Transactions on Circuits and Systems 28(3) (1981), 247-253.

[19] T. Faria, Stability and bifurcation for a delayed predator for a delayed predator-prey model and effect of diffusion, J. Math. Anal. Appl. 63 (2001), 254-433.

[20] X. Liao and G Chen, Local stability, Hopf and resonant codimension-two bifurcation in a harmonic oscillator with two time delays, Int J. Bifurcat. Chaos 11 (2001), 2105-2121.

[21] X. Meng and J. wei, Stability and bifurcation of mutual system with time delay, Chaos, Solitons \& Fractals 21 (2004), 729-740.

[22] S. Yuan and M. Han, Bifurcation analysis of a chemostat model with two distributed delays, Chaos, Solitons \& Fractals 20 (2004), 995-1004.

[23] M.S. Fofance and P.B. Ryba, Parametric stability of nonlinear time delay equations, International Journal of Non-Linear Mechanics 23(1) (2004), 79-91.

[24] A. Maccari, Approximate solution of a class of nonlinear oscillators in resonance with a periodic excitation, Nonlinear Dynamics $\mathbf{1 5}$ (1998), 329-343.

[25] A.F. El-Bassiouny and H.M. Abdelhafez, Predicition of bifurcations for external and parametric excited one-degree-of-freedom system with quadratic, cubic and quartic non-linearities, Mathematics and Computers in Simulation 57 (2001), 61-80.

[26] A.F. El-Bassiouny and M. Eissa, Dynamics of a single-degree-of-freedom structure with quadratic, cubic and quartic non-linearities to a harmonic resonance, Applied Mathematics and Computation 139 (2003), 1-21.

[27] A.R.F. Elhefnawy and A.F. El-Bassiouny, Non-linear stability and chaos in electrohydrodynamics, Chaos, Solitons \& Fractals 23 (2004), 289-312.

[28] A.F. El-Bassiouny, Principal parametric resonances of nonlinear mechanical system with two-frequency and self-excitations, Mechanics Research Communications, (2004), in press.

[29] A.M. Elnaggar and A.F. El-bassiouny, Harmonic, subharmonic, superharmonic, simul-taneous sub/super-harmonic and combination resonances of self-excited second order system to multi-frequency excitations, Acta Mechanica Sinca 8(1) (1993), 61-71.

[30] A. Elnaggar and A.F. El-bassiouny, Harmonic resonances of non-linear system of rods to a harmonic excitation, First International Conference of Mathematical and Physical Applications, Cairo, 1996.

[31] A. Maccari, Modulated motion and infinite-period bifurcation for two nonlinearly coupled and parametrically excited van der Pol oscillators, International Journal of Non-linear Mechanics 36 (2001), 335-347.

[32] A.F. El-Bassiouny and M. Eissa, Resonance of non-linear systems subjected to multi-parametrically excited structures: (comparison between two methods, response and stability), Physica Scripta 70 (2004), 101-113.

[33] A.F. El-Bassiouny, Effect of non-linmearities in elastomeric material dampers on torsional oscillation control, Applied Mathematics and Computations 162 (2004), 835-854.

[34] Q. Bi, Dynamical analysis of two coupled parametrically excited Van der pol oscillators, International Journal of Non-Linear Mechanics 39(1) (2004), 33-54. 

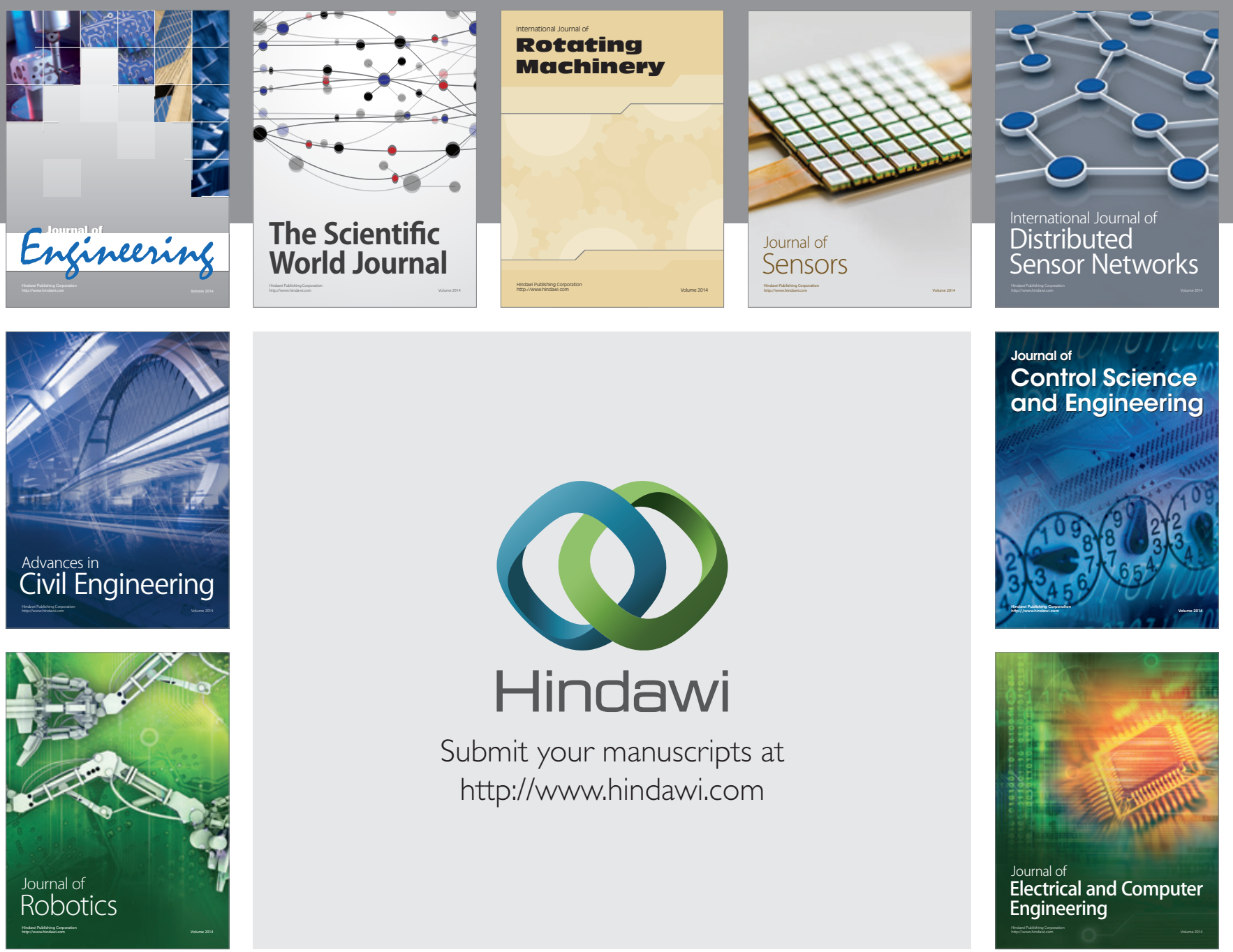

Submit your manuscripts at

http://www.hindawi.com
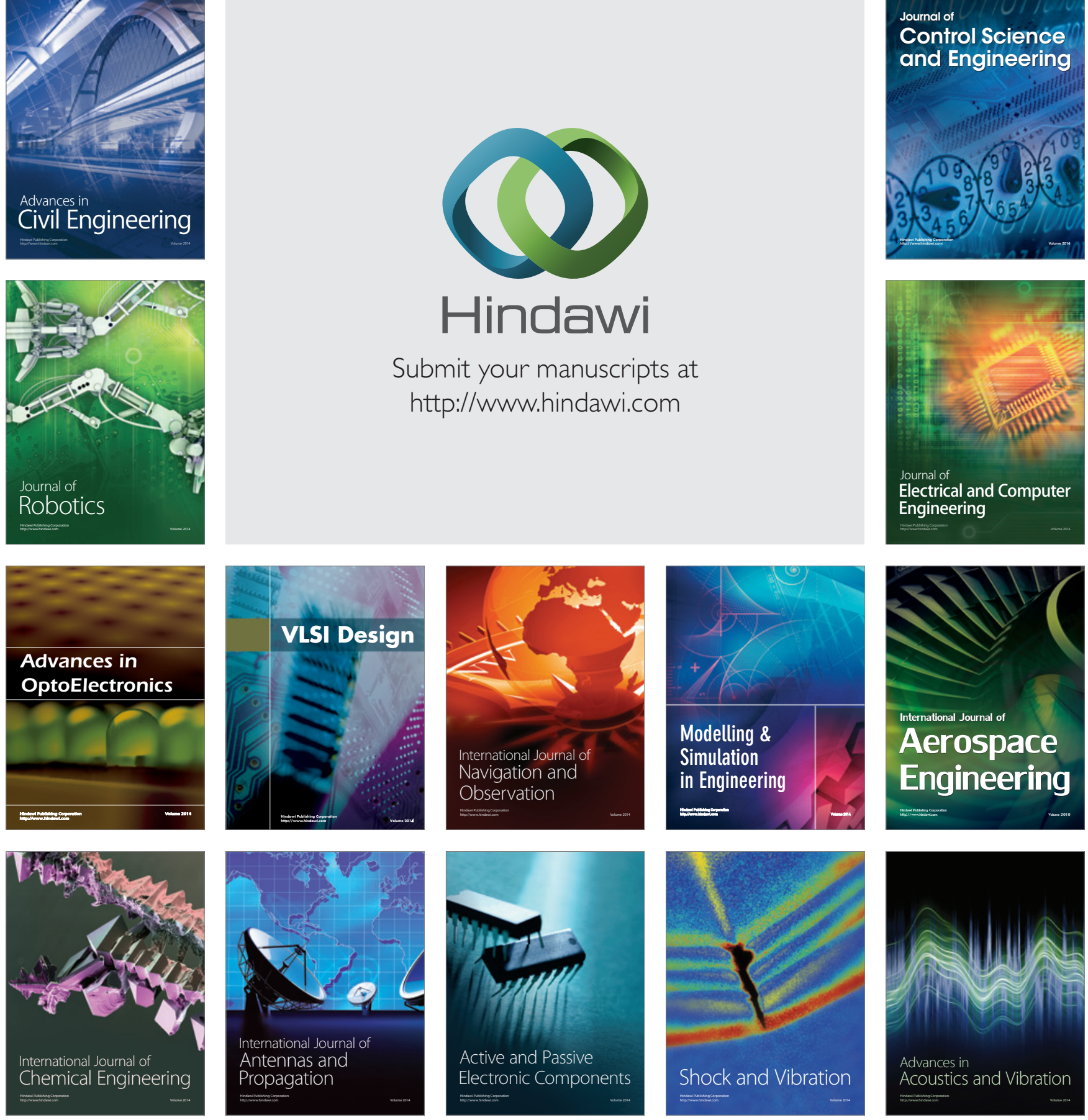